\title{
Acoustic Shock
}

National Cancer Institute

\section{Source}

National Cancer Institute. Acoustic Shock. NCI Thesaurus. Code C50452.

Neurophysiological and psychological symptoms caused by sudden and unexpected loud noise. 